ECCOMAS

Proceedia
COMPDYN 2017

$6^{\text {th }}$ ECCOMAS Thematic Conference on Computational Methods in Structural Dynamics and Earthquake Engineering M. Papadrakakis, M. Fragiadakis (eds.) Rhodes Island, Greece, 15-17 June, 2017

\title{
QUANTIFICATION OF GUIDED WAVE INTERACTION EFFECTS WITH LOCALIZED STRUCTURAL NONLINEARITIES IN COMPLEX, COMPOSITE STRUCTURES
}

\author{
V. Thierry ${ }^{1}$, D. Chronopoulos $^{1}$ and I. Ashcroft ${ }^{2}$ \\ ${ }^{1}$ Institute for Aerospace Technology (IAT) \\ The University of Nottingham, Innovation Park, NG7 2TU, UK \\ \& The Composites Group \\ The University of Nottingham, NG7 2RD, UK \\ ${ }^{2}$ Faculty of Engineering \\ The University of Nottingham, NG7 2RD, UK \\ e-mail: \{Victor.Thierry,Dimitrios.Chronopoulos,Ian.Ashcroft\}@ nottingham.ac.uk
}

Keywords: Structural nonlinearities, Wave Interaction with Damage, Finite Elements, Composite Structures, Nonlinear Ultrasound

\begin{abstract}
The extensive usage of composite materials in modern industrial applications implies a great range of possible structural failure modes for which the structure has to be frequently and thoroughly inspected. Nonlinear guided wave inspection techniques have been continuously gaining attention during the last decade. This is primarily due to their sensitivity to very small sizes of localized damage. A number of complex transformation phenomena take place when an elastic wave impinges on a nonlinear segment, including the generation of higher and sub-harmonics. Moreover, the transmission and reflection coefficients of each wave type become amplitude dependent. In this work, a generic Finite Element (FE) based computational scheme is presented for quantifying guided wave interaction effects with Localized Structural Nonlinearities (LSN) within structures of arbitrary layering and geometric complexity. Amplitude dependent guided wave reflection, transmission and conversion is computed through a Wave and Finite Element (WFE) method. The scheme couples wave propagation properties within linear structural waveguides to a LSN and is able to compute the generation of higher and sub-harmonics for each wave type through a harmonic balance projection. A Newton-like iteration scheme is employed for solving the system of nonlinear differential equations. $\mathrm{Nu}$ merical case studies are exhibited for a set of waveguides coupled through a joint exhibiting nonlinear mechanical behaviour.
\end{abstract}




\section{INTRODUCTION}

Modern industrial structures are increasingly made of composite layered materials due to their well-known benefits. Composite structures may however exhibit a great variety of structural failure modes (including delamination, fibre breakage, matrix cracking and debonding) and must be frequently inspected in order to ensure continuous structural integrity. An increasing tendency within the Structural Health Monitoring (SHM) community is the 'shift to the left' maintenance strategy [1] for which the earliest possible detection of damage is important. When it comes to the aeronautical industry, approximately $27 \%$ of an average modern aircraft's lifecycle cost [2] is spent on inspection and repair. The use of 'offline' structural inspection techniques currently leads to a massive reduction of the aircraft's availability and significant financial losses for the operator. The nondestructive detection and evaluation of damage in industrial structural components while operation is of paramount importance for monitoring the condition and residual life estimation of in-service structures. Linear ultrasonic Guided Wave $(\mathrm{GW})$ techniques have been widely employed for this purpose. These techniques however are primarily sensitive to gross defects but much less sensitive to micro-damage. Nonlinear acoustoultrasonic techniques, have been steadily receiving increasing attention during the last decade. Complex wave phenomena such as higher and subharmonic wave generation, nonlinear resonances or mixed frequency response can be induced by the two principal sources of nonlinearity in the structural system, namely nonlinear elasticity and contact nonlinearity.

Elastic wave distortion and generation of higher harmonics during propagation in nonlinear media has been reported as early as in [3]. The first attempt for modelling wave interaction with nonlinear joints can be found in $[4,5]$. It has been widely demonstrated that nonlinear ultrasonic techniques can be successfully deployed for detecting cracks as well as distributed structural deterioration (e.g. fatigue) $[6,7,8,9,10,11,12]$. The success of the developed methods is based on predicting and measuring the nonlinearities-induced wave effects which are pronounced in damaged and degraded structures but nearly un-measurable in the undamaged ones. A number of Nonlinear Elastic Wave Spectroscopy (NEWS) approaches [13, 14, 15] have also been presented and proved capable of detecting the presence of damage of very small sizes (in the order of $0.1 \mathrm{~mm}$ ) in composite structures. Wave propagation and material degradation detection in 1-D and 2-D media was investigated through a spring model in $[16,17]$. A numerical scheme for predicting nonlinear wave interaction with an interface of rough surfaces in contact was presented in [18]. The non-collinear mixing of bulk shear waves investigated in [19] presented significant potential for assessing material state than other nonlinear ultrasonic techniques because system nonlinearities can be both independently measured and largely eliminated. The development of an analytical framework for modelling the multi-modal guided wave interaction with damage was presented by the authors of [20,21]. In [22], a numerical scheme was presented in order to quantify the amplitude of the reflected compression and Rayleigh waves when impinging at the edge of an elastic plate. In [23] the authors coupled linear to nonlinear FE segments and investigated wave interaction with damage in 3-D solid media by means of a Landau's theory. Detection of fatigue damage in composite structures through higher harmonics generations has also recently been reported [24]. The short reviews provided by the authors in $[25,26]$ are informative on the general progress of nonlinear ultrasonics, while a comprehensive outline on the techniques dedicated to predicting and measuring higher harmonic generation in metallic structures is presented in [27]. An inclusive review on modelling wave-crack nonlinear interaction phenomena can be found in [28]. Despite the aforementioned attempts to capture wave interaction with LSNs, there is currently no generic computational scheme for predicting 
these quantities for composite structures of arbitrary layering and geometric complexity.

The FE based wave propagation analysis within periodic structures was firstly considered in [29]. The wave dispersion characteristics within the layered media can be accurately predicted for a very wide frequency range, by solving a polynomial eigenvalue problem for the propagation constants to be sought. The work was extended to 2-D media in [30]. The WFE method was introduced in [31] in order to facilitate the post-processing of the eigenproblem solutions and further improve the computational efficiency of the method. The vibration of a uniform waveguide through the WFE technique was investigated in $[32,33]$. The WFE method has recently found applications in predicting the vibroacoustic and dynamic performance of composite panels and shells [34, 35, 36, 37, 38, 39, 40], with pressurized shells [41, 42] and complex periodic structures $[43,44,45,46]$ having been investigated. The variability of acoustic transmission through layered structures [47, 48], as well as wave steering effects in anisotropic composites [49] have been modelled through the same methodology . The same FE based approach has been employed in order to compute the reflection and transmission coefficients of waves impinging on linear joints of finite dimensions [50, 51].

In this work, a generic FE-based computational scheme for computing wave interaction with LSNs is presented for the first time. Guided wave reflection, transmission and conversion is computed through a wave and finite element approach. The scheme couples wave propagation properties within linear structural waveguides to a LSN and is able to compute the generation higher and sub-harmonics for each wave type through a harmonic balance projection. The new approach is able to predict reflections and transmissions at harmonic frequencies with a speed that is orders of magnitude faster than conventional transient FE solutions. The structure can be of arbitrary complexity, layering and material characteristics as FE modelling is employed. A Galerkin projection is used to transform the system of nonlinear differential equations of motion into a set of nonlinear algebraic equations which is subsequently solved through a Newton's iteration method. The generation of wave harmonics, as well as amplitude-dependent wave reflection and transmission coefficients are reported through the exhibited numerical case studies. This is the first approach that can accurately and efficiently map the frequency-dependent interactions of guided waves with nonlinearities in arbitrarily complex structures.

The paper is organized as follows: In Sec.2 the formulation of the wave and finite element method for predicting acoustic and ultrasonic GW interaction with LSNs is presented. A description of the Galerkin projection as well as of the Newton's iteration scheme employed for solving the system of nonlinear differential equations is also given. In Sec.3 the proposed method is validated for a number of waveguide types through comparison to full transient FE analyses. Conclusions on the presented work are given in Sec.4.

\section{ELASTIC WAVE INTERACTION WITH STRUCTURAL NONLINEARITIES}

An arbitrarily complex and periodic in the $x$ direction waveguide is illustrated in Fig.2. The propagation constants for the elastic waves travelling in the $x$ direction within the waveguide can be sought through a WFE scheme. In the general case, we are assuming a system of $N$ waveguides connected through a structural coupling element which could exhibit arbitrarily complex mechanical behaviour and which is fully FE modelled (see Fig.2). As already stated, each waveguide can be of different and arbitrary layering and can also support a different number $W_{n}$ of propagating waves at a given frequency. An extensive description of how to deal with waveguides having different layouts and meshes can be found in [50]. Each supported 


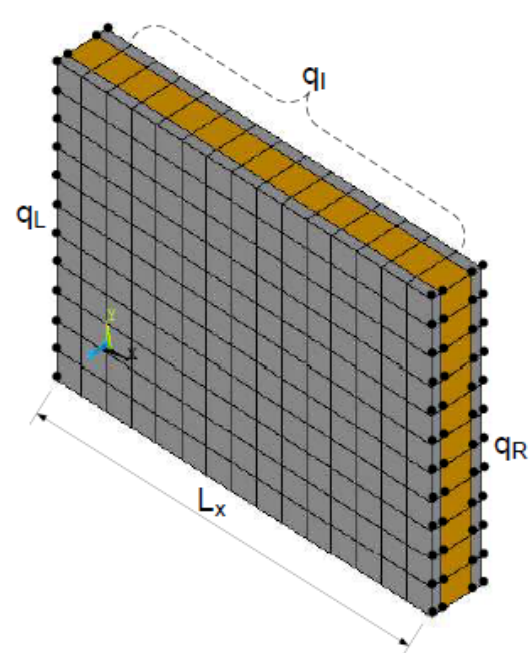

Figure 1: Caption of the WFE modelled composite waveguide with the left and right side nodes $\mathbf{q}_{L}, \mathbf{q}_{R}$ bullet marked. The range of interior nodes $\mathbf{q}_{I}$ is also illustrated.

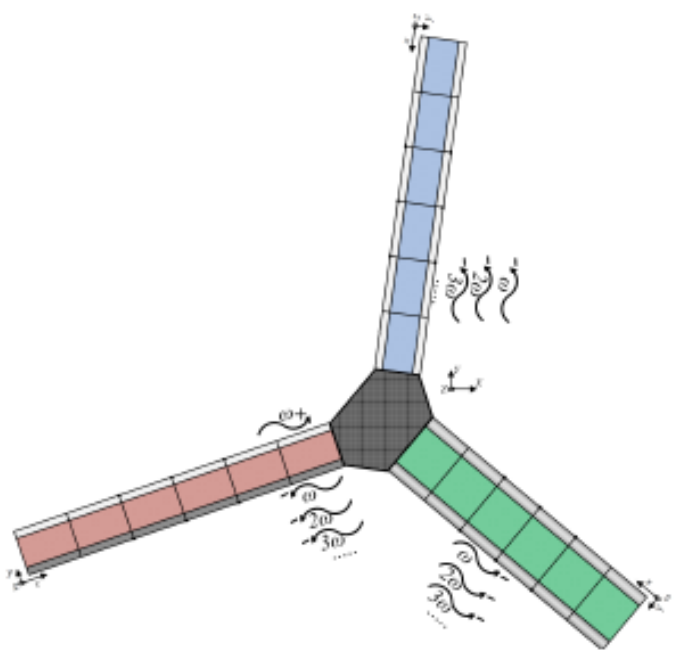

Figure 2: Periodic elastic waveguides connected through a coupling element exhibiting inelastic mechanical behaviour. A positive going wave ${ }^{+}$of angular frequency $\omega$ impinging on the coupling element will give rise to reflected and transmitted outgoing - waves of multiple subharmonic and superharmonic frequencies. 
wavemode $w$ with $w \in[1 \cdots W]$ for the $n$th waveguide in the system can be grouped as

$$
\begin{aligned}
& \boldsymbol{\Phi}_{n, q}^{h \omega,+}=\left[\begin{array}{llll}
\boldsymbol{\phi}_{q, 1}^{h \omega,+} & \phi_{q, 2}^{h \omega,+} & \cdots & \boldsymbol{\phi}_{q, W}^{h \omega,+}
\end{array}\right] \\
& \boldsymbol{\Phi}_{n, f}^{h \omega,+}=\left[\begin{array}{llll}
\boldsymbol{\phi}_{f, 1}^{h \omega,+} & \boldsymbol{\phi}_{f, 2}^{h \omega,+} & \cdots & \boldsymbol{\phi}_{f, W}^{h \omega,+}
\end{array}\right] \\
& \boldsymbol{\Phi}_{n, q}^{h \omega,-}=\left[\begin{array}{llll}
\boldsymbol{\phi}_{q, 1}^{h \omega,-} & \boldsymbol{\phi}_{q, 2}^{h \omega,-} & \cdots & \boldsymbol{\phi}_{q, W}^{h \omega,-}
\end{array}\right] \\
& \boldsymbol{\Phi}_{n, f}^{h \omega,-}=\left[\begin{array}{llll}
\boldsymbol{\phi}_{f, 1}^{h \omega,-} & \boldsymbol{\phi}_{f, 2}^{h \omega,-} & \cdots & \boldsymbol{\phi}_{f, W}^{h \omega,-}
\end{array}\right]
\end{aligned}
$$

with each matrix being of dimension $[j \times W]$. The wavemodes of the entire system can be computed for each waveguide $n$ with $n \in[1 \cdots N]$ and for the fundamental excitation frequency $\omega$ as well as for the higher and sub-harmonics $h \omega$ with $h \in\left[1 / H_{m} \cdots 1 \cdots H\right]$ and be grouped as

$$
\boldsymbol{\Phi}_{q}^{h \omega,+}=\left[\begin{array}{cccc}
\boldsymbol{\Phi}_{1, q}^{h \omega,+} & \mathbf{0} & \ldots & \mathbf{0} \\
\mathbf{0} & \boldsymbol{\Phi}_{2, q}^{h \omega,+} & \ldots & \mathbf{0} \\
\ldots & \ldots & \ldots & \ldots \\
\mathbf{0} & \mathbf{0} & \ldots & \boldsymbol{\Phi}_{N, q}^{h \omega,+}
\end{array}\right]_{[j N \times W N]}
$$

with similar expressions standing for $\boldsymbol{\Phi}_{f}^{h \omega,+}, \boldsymbol{\Phi}_{q}^{h \omega,-}, \boldsymbol{\Phi}_{f}^{h \omega,-}$. For each waveguide, the local coordinate system is defined such that the waveguide's axis is directed towards the joint (direction + ). The rotation matrix $\mathbf{R}_{n}$ transforms the DoFs from the local to the global coordinates of the system. Rotation matrices can be grouped in a block diagonal matrix $\mathbf{R}$. The equation of motion for the nonlinear, FE modelled coupling element can be in general written as

$$
\mathbb{M} \ddot{\mathbf{z}}(t)+\mathbb{C} \dot{\mathbf{z}}(t)+\mathbb{K} \mathbf{z}(t)+\mathbf{f}_{N L}=\mathbf{f}_{\text {ext }}(t)
$$

with $\mathbf{f}_{N L}$ the nonlinear force vector induced by the coupling element's inherent inelastic behaviour and $\mathbf{f}_{\text {ext }}(t)$ being the external forces applied to the coupling element.

Waves of the fundamental excitation frequency $\omega$ having amplitudes $\mathbf{a}_{n}^{\omega,+}$ are impinging on the coupling element from the $n$th waveguide. For each considered harmonic $h \omega$, they give rise to reflected waves of amplitudes $\mathbf{a}_{n}^{h \omega,-}=\mathbf{r}_{n, n}^{h \omega} \mathbf{a}_{n}^{\omega,+}$ in the $n$th waveguide, while they also give rise to transmitted waves of amplitudes $\mathbf{a}_{k}^{h \omega,-}=\mathbf{c}_{k, n}^{h \omega} \mathbf{a}_{n}^{\omega,+}$ in the $k$ th waveguide with $\mathbf{r}_{n, n}^{h \omega}$ and $\mathbf{c}_{k, n}^{h \omega}$ being matrices containing the reflection and transmission coefficients of the coupling element at each harmonic $h \omega$. These define the wave scattering matrix $\mathbf{S}^{h \omega}$ of the joint, whose partitions relate the amplitudes of the incident and scattered waves as

$$
\mathbf{a}^{h \omega,-}=\mathbf{S}^{h \omega} \mathbf{a}^{\omega,+}
$$

with $\mathbf{a}_{[W N \times 1]}^{\omega,+}$ the vector containing the amplitudes of the incoming waves moving towards the coupling element and $\mathbf{a}_{[W N \times 1]}^{h \omega,-}$ the vector containing the amplitudes of the reflected and transmitted outgoing waves produced at the $h \omega$ harmonic. The complex wave interaction matrix $\mathbf{S}^{h \omega}$ projects the incoming waves $\mathbf{a}^{\omega,+}$ having a single frequency $\omega$ to each of the higher and sub-harmonics $h \omega$. Assuming that only waves of a single frequency $\omega$ are impinging on the coupling element, a transformation can be defined for the motion in the waveguides between the physical domain, where the motion is described in terms of $\mathbf{q}$ and $\mathbf{f}$ and the wave domain where the motion is described in terms of waves of amplitudes $\mathbf{a}^{\omega,+}$ and $\mathbf{a}^{h \omega,-}$ travelling in the positive and negative directions respectively, and by concatenating the corresponding vectors 
and matrices the general expressions for $\mathbf{q}$ and $\mathbf{f}$ for the system of waveguides can be expressed as

$$
\begin{aligned}
& \mathbf{q}(t)=\boldsymbol{\Phi}_{q}^{\omega,+} \mathbf{a}^{+} \cos (\omega t)+\sum_{h=1}^{H} \boldsymbol{\Phi}_{q}^{h \omega,-} \mathbf{a}^{h \omega,-} \cos (h \omega t)+\sum_{h=1}^{H_{m}} \boldsymbol{\Phi}_{q}^{h \omega,-} \mathbf{a}^{h \omega,-} \cos \left(\frac{1}{h} \omega t\right) \\
& \mathbf{f}(t)=\boldsymbol{\Phi}_{f}^{\omega,+} \mathbf{a}^{+} \cos (\omega t)+\sum_{h=1}^{H} \boldsymbol{\Phi}_{f}^{h \omega,-} \mathbf{a}^{h \omega,-} \cos (h \omega t)+\sum_{h=1}^{H_{m}} \boldsymbol{\Phi}_{f}^{h \omega,-} \mathbf{a}^{h \omega,-} \cos \left(\frac{1}{h} \omega t\right)
\end{aligned}
$$

The above expression can be employed to perform a cyclostationary projection of the behaviour of the system through the Harmonic Balance Method (HBM) [52]. It is noted that sin terms are not included in the above expansions as the phase of each wave scattering coefficient is captured by the imaginary part of the sought interaction coefficients $\mathbf{a}^{h \omega,-}$. By performing the convenient substitution $\tau=\omega t$ and grouping the trigonometric terms the following expression can be acquired

$$
\mathbf{q}(\tau)=\mathbf{\Phi}_{q}^{\omega,+} \mathbf{T}_{2} \mathbf{a}^{+}+\mathbf{T}_{1} \mathcal{A}_{q}^{\omega,-}
$$

with $\mathcal{A}_{q}^{\omega,-}$ being the generalized displacement wave interaction coefficient vector written as

$$
\mathcal{A}_{q}^{\omega,-}=\left\{\begin{array}{c}
\boldsymbol{\Phi}_{q}^{\omega,-} \mathbf{S}^{\omega} \mathbf{a}^{\omega,+} \\
\boldsymbol{\Phi}_{q}^{2 \omega,-} \mathbf{S}^{2 \omega} \mathbf{a}^{\omega,+} \\
\cdots \\
\boldsymbol{\Phi}_{q}^{H \omega,-} \mathbf{S}^{H \omega} \mathbf{a}^{\omega,+} \\
\cdots \\
\boldsymbol{\Phi}_{q}^{\frac{1}{H_{m}} \omega,-} \mathbf{S}^{\frac{1}{H_{m}} \omega} \mathbf{a}^{\omega,+}
\end{array}\right\}_{[j H N \times 1]}
$$

$\mathrm{q}(\tau)$ can be derived twice. Then with $\mathcal{A}_{f}^{\omega,-}$ formulated similarly to $\mathcal{A}_{q}^{\omega,-}$. A set of nonlinear, algebraic equations can be obtained through a Galerkin projection [53] of the system of nonlinear differential equations of motion.

$$
\begin{aligned}
& \int_{0}^{2 H_{m} \pi}\left[\mathbf{T}_{1}^{\top}\left[\omega^{2} \mathbb{M} \mathbf{R} \frac{\mathrm{d}^{2} \mathbf{T}_{1}}{\mathrm{~d} \tau^{2}}+\omega \mathbb{C} \mathbf{R} \frac{\mathrm{d} \mathbf{T}_{1}}{\mathrm{~d} \tau}+\mathbb{K} \mathbf{R} \mathbf{T}_{1}\right] \mathcal{A}_{q}^{\omega,-}-\mathbf{T}_{1}^{\top} \mathbf{R} \mathbf{T}_{1} \mathcal{A}_{f}^{\omega,-}\right] \mathrm{d} \tau+ \\
& \int_{0}^{2 H_{m} \pi} \mathbf{T}_{1}^{\top}\left[\omega^{2} \mathbf{M} \mathbf{R} \Phi_{q}^{\omega,+} \frac{\mathrm{d}^{2} \mathbf{T}_{2}}{\mathrm{~d} \tau^{2}}+\omega \mathbb{C R} \boldsymbol{\Phi}_{q}^{\omega,+} \frac{\mathrm{d} \mathbf{T}_{2}}{\mathrm{~d} \tau}+\mathbb{K} \mathbf{R} \boldsymbol{\Phi}_{q}^{\omega,+} \mathbf{T}_{2}-\mathbf{R} \boldsymbol{\Phi}_{f}^{\omega,+} \mathbf{T}_{2}\right] \mathbf{a}^{+} \mathrm{d} \tau+ \\
& \int_{0}^{2 H_{m} \pi} \mathbf{T}_{1}^{\top} \mathbf{f}_{N L} \mathrm{~d} \tau=\mathbf{0}
\end{aligned}
$$

\section{VALIDATION FOR A LAYERED COMPOSITE BEAM}

The presented approach is validated for an asymmetric layered composite beam with aluminium facesheets and a polyurethane core having a cross-section of $8 \mathrm{~mm} \times 12 \mathrm{~mm}$ and the thicknesses of the layers being equal to $1 \mathrm{~mm}, 10 \mathrm{~mm}$ and $2 \mathrm{~mm}$ respectively (see Fig.3). The entirety of the propagating waves can be sought through WFE and without the need of any kinematic assumptions for the complex structures, as 3D FEs and displacement fields are employed.

In practice, the wave modes can be excited one-by-one in a full FE transient simulation by employing the WFE computed $\phi_{q, n}^{+}$eigenvectors and applying them as time-dependent harmonic displacement boundary conditions (of excitation frequency $\omega$ ) at one of the extreme cross-sections of the waveguide. An 11-period Hanning window was used for all transient excitations. The results on the fundamental and harmonic reflections for a nonlinearly hardening 


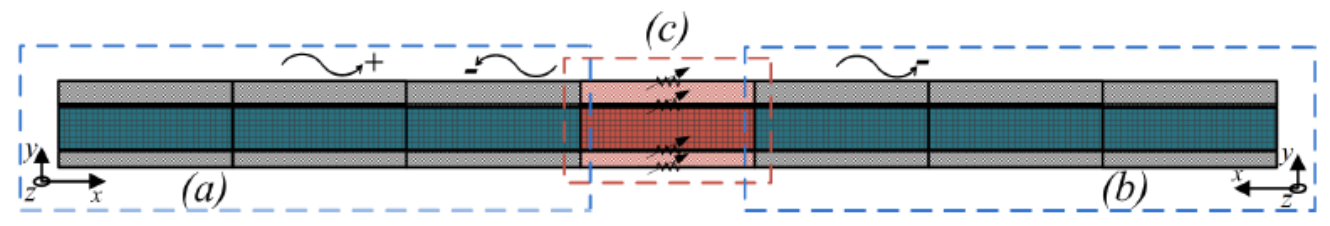

Figure 3: Schematic representation of the two healthy and elastic composite multilayer waveguides (a) and (b) coupled through a nonlinear element (c) containing an arbitrary structural nonlinearity.

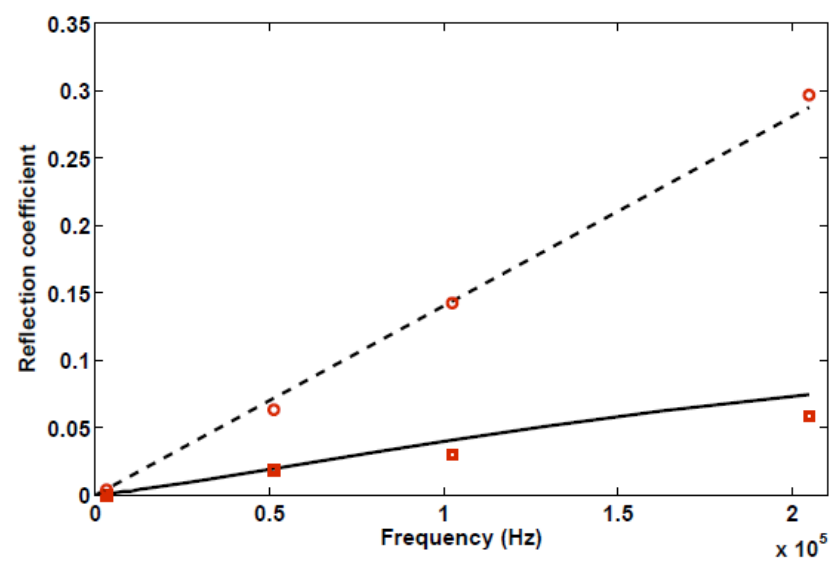

Figure 4: Absolute values of the wave reflection coefficients of the pressure propagating wave for a nonlinearly hardened element with $k_{n l}=1 \mathrm{e} 13 \mathrm{~N} / \mathrm{m}^{3}$ at: Frequency $\omega$ computed according to the current scheme (- $)$ and through a full transient FE calculation (o), Harmonic frequency $3 \omega$ computed according to the current scheme (-) and through a full transient FE calculation ( $\square$ ).

element are presented below in Figs.4. Excellent agreement is observed between the fundamental frequency reflection predictions obtained through the presented approach and through a full FE transient response prediction. Moreover, good agreement is observed between the two approaches regarding the reflections computed for the $3 \omega$ harmonic frequency. The most probable cause of the slight divergence observed between the two sets of results is the fact that in a nonlinear transient FE problem energy is partially also channeled towards other harmonics (higher than the second one), which are not included in the harmonic balance expansion.

\section{CONCLUSIONS}

A novel comprehensive FE-based computational scheme was presented for quantifying guided wave interaction with LSNs. Structures of arbitrary complexity, layering and material characteristics can be modelled through the presented approach as an FE discretization is employed. The scheme couples wave propagation properties within linear structural waveguides to LSNs and is able to compute the generation of harmonic frequencies for each wave mode through a harmonic balance projection. The principal outcomes of the work are summarized as follows:

- The presented scheme was validated through comparison with a full FE transient response prediction. Excellent agreement is observed between the two sets of results for the fundamental, as well as for higher harmonic frequency predictions.

- The new approach is able to predict reflections and transmissions at harmonic frequencies with a speed that is orders of magnitude faster than conventional transient FE solutions. 
- Generation of higher order harmonics can become maximum at certain frequencies which can be excited for facilitating the detection of certain nonlinearity scenarios (ideally related to the presence of certain damage).

Future developments are focusing towards modelling and implementing realistic damage models as a LSN. Efficient multiscale damage models are essential to implement in order to accurately capture the nonlinear mechanics of advanced damage scenarios, while retaining the size of the FE model and the implied required computational effort at acceptable levels.

\section{ACKNOWLEDGMENTS}

This work is funded by the INNOVATIVE doctoral programme. The INNOVATIVE programme is partially funded by the Marie Curie Initial Training Networks (ITN) action (project number 665468) and partially by the Institute for Aerospace Technology (IAT) at the University of Nottingham. 


\section{REFERENCES}

[1] C. Lissenden, Y. Liu, and J. Rose, "Use of non-linear ultrasonic guided waves for early damage detection," Insight-Non-Destructive Testing and Condition Monitoring, vol. 57, no. 4, pp. 206-211, 2015.

[2] S. S. Kessler, S. M. Spearing, and C. Soutis, "Damage detection in composite materials using lamb wave methods," Smart Materials and Structures, vol. 11, no. 2, pp. 269-, 2002.

[3] M. Breazeale and D. Thompson, "Finite-amplitude ultrasonic waves in aluminum," Applied Physics Letters, vol. 3, no. 5, pp. 77-78, 1963.

[4] A. Vakakis, "Scattering of structural waves by nonlinear elastic joints," Journal of vibration and acoustics, vol. 115, no. 4, pp. 403-410, 1993.

[5] A. H. Nayfeh, A. F. Vakakis, and T. A. Nayfeh, "A method for analyzing the interaction of nondispersive structural waves and nonlinear joints," The Journal of the Acoustical Society of America, vol. 93, no. 2, pp. 849-856, 1993.

[6] P. B. Nagy, "Fatigue damage assessment by nonlinear ultrasonic materials characterization," Ultrasonics, vol. 36, no. 1, pp. 375-381, 1998.

[7] D. M. Donskoy and A. M. Sutin, "Vibro-acoustic modulation nondestructive evaluation technique," Journal of intelligent material systems and structures, vol. 9, no. 9, pp. 765$771,1998$.

[8] V. Zaitsev, V. Nazarov, V. Gusev, and B. Castagnede, "Novel nonlinear-modulation acoustic technique for crack detection," NDT \& E International, vol. 39, no. 3, pp. 184-194, 2006.

[9] V. J. Rao, E. Kannan, R. V. Prakash, and K. Balasubramaniam, "Fatigue damage characterization using surface acoustic wave nonlinearity in aluminum alloy aa7175-t7351," Journal of Applied Physics, vol. 104, no. 12, pp. 123508-, 2008.

[10] H. Hu, W. Staszewski, N. Hu, R. Jenal, and G. Qin, "Crack detection using nonlinear acoustics and piezoceramic transducersinstantaneous amplitude and frequency analysis," Smart Materials and Structures, vol. 19, no. 6, pp. 065017-, 2010.

[11] Z. Su, C. Zhou, M. Hong, L. Cheng, Q. Wang, and X. Qing, "Acousto-ultrasonics-based fatigue damage characterization: Linear versus nonlinear signal features," Mechanical Systems and Signal Processing, vol. 45, no. 1, pp. 225-239, 2014.

[12] H. J. Lim, H. Sohn, M. P. DeSimio, and K. Brown, "Reference-free fatigue crack detection using nonlinear ultrasonic modulation under various temperature and loading conditions," Mechanical Systems and Signal Processing, vol. 45, no. 2, pp. 468-478, 2014.

[13] K.-A. Van Den Abeele, P. A. Johnson, and A. Sutin, "Nonlinear elastic wave spectroscopy (news) techniques to discern material damage, part i: nonlinear wave modulation spectroscopy (nwms)," Research in nondestructive evaluation, vol. 12, no. 1, pp. 17-30, 2000. 
[14] K. E. Van Den Abeele, A. Sutin, J. Carmeliet, and P. A. Johnson, "Micro-damage diagnostics using nonlinear elastic wave spectroscopy (news)," Ndt \& E International, vol. 34, no. 4, pp. 239-248, 2001.

[15] M. Meo, U. Polimeno, and G. Zumpano, "Detecting damage in composite material using nonlinear elastic wave spectroscopy methods," Applied composite materials, vol. 15, no. 3, pp. 115-126, 2008.

[16] M. Scalerandi, V. Agostini, P. P. Delsanto, K. Van Den Abeele, and P. A. Johnson, "Local interaction simulation approach to modelling nonclassical, nonlinear elastic behavior in solids," The Journal of the Acoustical Society of America, vol. 113, no. 6, pp. 3049-3059, 2003.

[17] P. P. Delsanto, A. Gliozzi, M. Hirsekorn, and M. Nobili, "A 2d spring model for the simulation of ultrasonic wave propagation in nonlinear hysteretic media," Ultrasonics, vol. 44, no. 3, pp. 279-286, 2006.

[18] C. Pecorari, "Nonlinear interaction of plane ultrasonic waves with an interface between rough surfaces in contact," The Journal of the Acoustical Society of America, vol. 113, no. 6, pp. 3065-3072, 2003.

[19] A. J. Croxford, P. D. Wilcox, B. W. Drinkwater, and P. B. Nagy, "The use of non-collinear mixing for nonlinear ultrasonic detection of plasticity and fatigue," The Journal of the Acoustical Society of America, vol. 126, no. 5, pp. EL117-EL122, 2009.

[20] V. Giurgiutiu, M. Gresil, B. Lin, A. Cuc, Y. Shen, and C. Roman, "Predictive modeling of piezoelectric wafer active sensors interaction with high-frequency structural waves and vibration," Acta Mechanica, vol. 223, no. 8, pp. 1681-1691, 2012.

[21] Y. Shen and V. Giurgiutiu, "Predictive modeling of nonlinear wave propagation for structural health monitoring with piezoelectric wafer active sensors," Journal of Intelligent Material Systems and Structures, vol. 25, no. 4, pp. 506-520, 2014.

[22] T. B. Autrusson, K. G. Sabra, and M. J. Leamy, "Reflection of compressional and rayleigh waves on the edges of an elastic plate with quadratic nonlinearity," The Journal of the Acoustical Society of America, vol. 131, no. 3, pp. 1928-1937, 2012.

[23] G. M. Fierro, F. Ciampa, D. Ginzburg, E. Onder, and M. Meo, "Nonlinear ultrasound modelling and validation of fatigue damage," Journal of Sound and Vibration, vol. 343, pp. 121-130, 2015.

[24] N. Rauter, R. Lammering, and T. Kühnrich, "On the detection of fatigue damage in composites by use of second harmonic guided waves," Composite Structures, vol. 152, pp. 247-258, 2016.

[25] K.-Y. Jhang, "Nonlinear ultrasonic techniques for nondestructive assessment of micro damage in material: a review," International journal of precision engineering and manufacturing, vol. 10, no. 1, pp. 123-135, 2009.

[26] I. Solodov, D. Döring, and G. Busse, "New opportunities for ndt using non-linear interaction of elastic waves with defects," Strojniški vestnik-Journal of Mechanical Engineering, vol. 57, no. 3, pp. 169-182, 2011. 
[27] K. Matlack, J.-Y. Kim, L. Jacobs, and J. Qu, "Review of second harmonic generation measurement techniques for material state determination in metals," Journal of Nondestructive Evaluation, vol. 34, no. 1, pp. 1-23, 2015.

[28] D. Broda, W. Staszewski, A. Martowicz, T. Uhl, and V. Silberschmidt, "Modelling of nonlinear crack-wave interactions for damage detection based on ultrasounda review," Journal of Sound and Vibration, vol. 333, no. 4, pp. 1097-1118, 2014.

[29] D. Mead, "A general theory of harmonic wave propagation in linear periodic systems with multiple coupling," Journal of Sound and Vibration, vol. 27, no. 2, pp. 235-260, 1973.

[30] R. Langley, "A note on the force boundary conditions for two-dimensional periodic structures with corner freedoms," Journal of Sound and Vibration, vol. 167, no. 2, pp. 377-381, 1993.

[31] B. R. Mace, D. Duhamel, M. J. Brennan, and L. Hinke, "Finite element prediction of wave motion in structural waveguides," The Journal of the Acoustical Society of America, vol. 117 , no. 5, pp. 2835-2843, 2005.

[32] D. Duhamel, B. R. Mace, and M. J. Brennan, "Finite element analysis of the vibrations of waveguides and periodic structures," Journal of Sound and Vibration, vol. 294, no. 1, pp. 205-220, 2006.

[33] J. M. Renno and B. R. Mace, "On the forced response of waveguides using the wave and finite element method," Journal of Sound and Vibration, vol. 329, no. 26, pp. 5474-5488, 2010.

[34] D. Chronopoulos, B. Troclet, M. Ichchou, and J. Laine, "A unified approach for the broadband vibroacoustic response of composite shells," Composites Part B: Engineering, vol. 43, no. 4, pp. 1837-1846, 2012.

[35] D. Chronopoulos, B. Troclet, O. Bareille, and M. Ichchou, "Modeling the response of composite panels by a dynamic stiffness approach," Composite Structures, vol. 96, pp. 111-120, 2013.

[36] D. Chronopoulos, M. Ichchou, B. Troclet, and O. Bareille, "Efficient prediction of the response of layered shells by a dynamic stiffness approach," Composite Structures, vol. 97 , pp. 401-404, 2013.

[37] D. Chronopoulos, M. Ichchou, B. Troclet, and O. Bareille, "Predicting the broadband vibroacoustic response of systems subject to aeroacoustic loads by a krylov subspace reduction," Applied Acoustics, vol. 74, no. 12, pp. 1394-1405, 2013.

[38] D. Chronopoulos, M. Ichchou, B. Troclet, and O. Bareille, "Thermal effects on the sound transmission through aerospace composite structures," Aerospace Science and Technology, vol. 30, no. 1, pp. 192-199, 2013.

[39] D. Chronopoulos, M. Ichchou, B. Troclet, and O. Bareille, "Predicting the broadband response of a layered cone-cylinder-cone shell," Composite Structures, vol. 107, pp. 149$159,2014$. 
[40] D. Chronopoulos, M. Ichchou, B. Troclet, and O. Bareille, "Computing the broadband vibroacoustic response of arbitrarily thick layered panels by a wave finite element approach," Applied Acoustics, vol. 77, pp. 89-98, 2014.

[41] V. Polenta, S. Garvey, D. Chronopoulos, A. Long, and H. Morvan, "Optimal internal pressurisation of cylindrical shells for maximising their critical bending load," Thin-Walled Structures, vol. 87, pp. 133-138, 2015.

[42] T. Ampatzidis and D. Chronopoulos, "Acoustic transmission properties of pressurised and pre-stressed composite structures," Composite Structures, vol. 152, pp. 900-912, 2016.

[43] I. Antoniadis, D. Chronopoulos, V. Spitas, and D. Koulocheris, "Hyper-damping properties of a stiff and stable linear oscillator with a negative stiffness element," Journal of Sound and Vibration, vol. 346, no. 1, pp. 37-52, 2015.

[44] D. Chronopoulos, M. Collet, and M. Ichchou, "Damping enhancement of composite panels by inclusion of shunted piezoelectric patches: A wave-based modelling approach," Materials, vol. 8, no. 2, pp. 815-828, 2015.

[45] D. Chronopoulos, I. Antoniadis, M. Collet, and M. Ichchou, "Enhancement of wave damping within metamaterials having embedded negative stiffness inclusions," Wave Motion, vol. 58, pp. 165-179, 2015.

[46] D. Chronopoulos, I. Antoniadis, and T. Ampatzidis, "Enhanced acoustic insulation properties of composite metamaterials having embedded negative stiffness inclusions," Extreme Mechanics Letters, 2016.

[47] D. Chronopoulos, "Design optimization of composite structures operating in acoustic environments," Journal of Sound and Vibration, vol. 355, pp. 322-344, 2015.

[48] M. Ben Souf, D. Chronopoulos, M. Ichchou, O. Bareille, and M. Haddar, "On the variability of the sound transmission loss of composite panels through a parametric probabilistic approach," Journal of Computational Acoustics, vol. 24, no. 1, 2016.

[49] D. Chronopoulos, "Wave steering effects in anisotropic composite structures: Direct calculation of the energy skew angle through a finite element scheme," Ultrasonics, vol. 73, pp. 43-48, 2017.

[50] J.-M. Mencik and M. Ichchou, "Multi-mode propagation and diffusion in structures through finite elements," European Journal of Mechanics-A/Solids, vol. 24, no. 5, pp. 877898, 2005.

[51] J. M. Renno and B. R. Mace, "Calculation of reflection and transmission coefficients of joints using a hybrid finite element/wave and finite element approach," Journal of Sound and Vibration, vol. 332, no. 9, pp. 2149-2164, 2013.

[52] R. E. Mickens, Truly nonlinear oscillations: harmonic balance, parameter expansions, iteration, and averaging methods. World Scientific, 2010.

[53] R. K. Narisetti, M. Ruzzene, and M. J. Leamy, "Study of wave propagation in strongly nonlinear periodic lattices using a harmonic balance approach," Wave Motion, vol. 49, no. 2, pp. 394-410, 2012. 